Check for updates

Cite this: Chem. Sci., 2018, 9, 8352

๑ All publication charges for this article have been paid for by the Royal Society of Chemistry

Received 19th March 2018

Accepted 4th September 2018

DOI: $10.1039 / c 8 s c 01284 a$

rsc.li/chemical-science

\section{Molecular mechanism of phosphoinositides' specificity for the inwardly rectifying potassium channel Kir2.2†}

\author{
Xuan-Yu Meng, $\ddagger^{\mathrm{a}}$ Seung-gu Kang: $t^{\mathrm{b}}$ and Ruhong Zhou (D) *abc
}

Phosphoinositides are essential signaling lipids that play a critical role in regulating ion channels, and their dysregulation often results in fatal diseases including cardiac arrhythmia and paralysis. Despite decades of intensive research, the underlying molecular mechanism of lipid agonism and specificity remains largely unknown. Here, we present a systematic study of the binding mechanism and specificity of a native agonist, phosphatidylinositol 4,5-bisphosphate $\left(\mathrm{PI}(4,5) \mathrm{P}_{2}\right)$ and two of its variants, $\mathrm{PI}(3,4) \mathrm{P}_{2}$ and $\mathrm{PI}(3,4,5) \mathrm{P}_{3}$, on inwardly rectifying potassium channel Kir2.2, using molecular dynamics simulations and free energy perturbations (FEPs). Our results demonstrate that the major driving force for the $\mathrm{PI}(4,5) \mathrm{P}_{2}$ specificity on Kir2.2 comes from the highly organized salt-bridge network formed between the charged inositol head and phosphodiester linker of $\mathrm{PI}(4,5) \mathrm{P}_{2}$. The unsaturated arachidonic chain is also shown to contribute to the stable binding through hydrophobic interactions with nearby Kir2.2 hydrophobic residues. Consistent with previous experimental findings, our FEP results confirmed that non-native ligands, $\mathrm{PI}(3,4) \mathrm{P}_{2}$ and $\mathrm{PI}(3,4,5) \mathrm{P}_{3}$, show significant loss in binding affinity as a result of the substantial shift from the native binding mode and unfavorable local solvation environment. However, surprisingly, the underlying molecular pictures for the unfavorable binding of both ligands are quite distinctive: for $\mathrm{PI}(3,4) \mathrm{P}_{2}$, it is due to a direct destabilization in the bound state, whereas for $\mathrm{PI}(3,4,5) \mathrm{P}_{3}$, it is due to a relative stabilization in its free state. Our findings not only provide a theoretical basis for the ligand specificity, but also generate new insights into the allosteric modulation of ligand-gated ion channels.

\section{Introduction}

Phosphoinositides (PIPs) are one class of signaling lipid molecules distributed broadly in the inner leaflet of the plasma membrane, which play critical roles in diverse physiological functions, such as regulating the activity of ion channels and transporters, endocytosis and exocytosis, and calcium signaling. ${ }^{1-6}$ The most abundant phosphoinositide in membranes is phosphatidylinositol 4,5-bisphosphate $\left(\mathrm{PI}(4,5) \mathrm{P}_{2}\right)$ whose well-known functional roles include the generation of two critical second messengers, inositol trisphosphate (IP3) and diacyl glycerol (DAG) after being hydrolyzed, as well as its broad regulation of almost all ion channels and many transporters. ${ }^{7-11}$ Structurally, $\operatorname{PI}(4,5) \mathrm{P}_{2}$ is composed of an inositol head group with two phosphates on its $4^{\prime}$

\footnotetext{
${ }^{a}$ State Key Laboratory of Radiation Medicine and Protection, School for Radiological and Interdisciplinary Sciences (RAD-X), Collaborative Innovation Centre of Radiation Medicine of Jiangsu Higher Education Institutions, Soochow University, Suzhou 215123, China

${ }^{b} I B M$ Thomas J. Watson Research Center, Yorktown Heights, NY 10598, USA. E-mail: ruhongz@us.ibm.com

'Department of Chemistry, Columbia University, New York, NY 10027, USA

$\dagger$ Electronic supplementary information (ESI) available. See DOI: $10.1039 / \mathrm{c} 8 \mathrm{sc} 01284 \mathrm{a}$

\$ These authors contributed equally.
}

and $5^{\prime}$ positions, linked by two fatty acid tails (i.e., stearic and arachidonic acids) through a phosphodiester linker in-between. Variations in the number and position of phosphorylation on the inositide group produce different derivatives, such as $\mathrm{PI}(3,4) \mathrm{P}_{2}$ and $\mathrm{PI}(3,4,5) \mathrm{P}_{3}$.

Since the early 1990 s, people realized that $\mathrm{PI}(4,5) \mathrm{P}_{2}$ directly interacts with the inwardly rectifying potassium (Kir) channels and controls the channel activity. ${ }^{12-14}$ Many studies focused on uncovering the molecular mechanism of $\mathrm{PI}(4,5) \mathrm{P}_{2}-$ Kir interactions. ${ }^{15-19}$ The binding of $\mathrm{PI}(4,5) \mathrm{P}_{2}$ upon Kir channels involves a very exquisite way: it locates at two different interfaces, with one at the vertical interface between the transmembrane and cytoplasmic domains, and the other at the horizontal interface between the adjacent subunits of the channel (more below in Fig. 1). Thus, one $\mathrm{PI}(4,5) \mathrm{P}_{2}$ may interact with two domains as well as two subunits simultaneously. The co-crystal structure of the chicken Kir2.2 with $\mathrm{PI}(4,5) \mathrm{P}_{2}$ indicates that $\mathrm{PI}(4,5) \mathrm{P}_{2}$ may induce the helix-formation of tether-helix in one subunit and Cterminus of the Slide helix in the adjacent subunit. ${ }^{18}$ The main driving force for $\mathrm{PI}(4,5) \mathrm{P}_{2}$ and Kir channels is the electrostatic attraction: $\mathrm{PI}(4,5) \mathrm{P}_{2}$ bears negative charges under the physiological conditions and the binding site of Kir complementarily consists of multiple positive charged residues. ${ }^{19}$ A series of important basic residues that form the salt bridges with $\mathrm{PI}(4,5)$ 

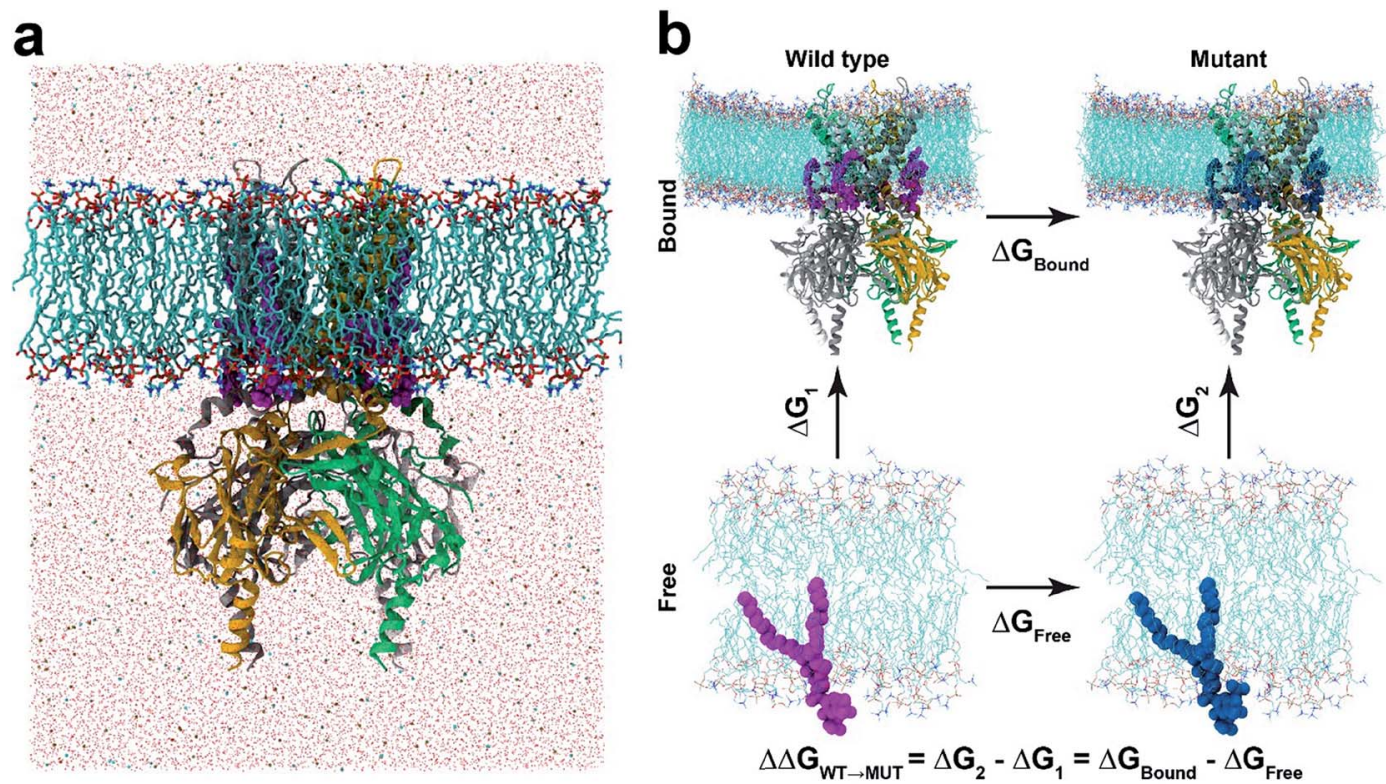

Fig. 1 Molecular dynamics and free energy perturbation simulation. (a) Molecular dynamics set-up. KIR2.2 bound with four PI(4,5)P 2 (marked in magenta) is embedded in a POPC membrane bilayer, as solvated with $150 \mathrm{mM} \mathrm{KCl}$. (b) Thermodynamic cycle for free energy perturbation calculations. The binding affinities of mutant phosphoinositides $\left(\mathrm{PI}(3,4,5) \mathrm{P}_{3}\right.$ and $\left.\mathrm{PI}(3,4) \mathrm{P}_{2}\right)$ are calculated relative to that of $\mathrm{PI}(4,5) \mathrm{P}_{2}$ by calculating FEPs for $\Delta G_{\text {bound }}$ and $\Delta G_{\text {free, }}$ where the wild-type $\left(\mathrm{PI}(4,5) \mathrm{P}_{2}\right)$ and mutants $\left(\mathrm{PI}(3,4,5) \mathrm{P}_{3}\right.$ or $\left.\mathrm{PI}(3,4) \mathrm{P}_{2}\right)$ are depicted in magenta and blue balls, respectively.

$\mathrm{P}_{2}$ were also indicated through electrophysiology and crystallography studies. ${ }^{17-19}$ Mutagenesis of these basic residues either decreases the channel sensitivity to $\mathrm{PI}(4,5) \mathrm{P}_{2}$ or damages the channel activity. ${ }^{19,20}$

Regarding the electrostatic interaction for the $\mathrm{PI}(4,5) \mathrm{P}_{2}-\mathrm{Kir}$ channel, it is interesting to raise the question whether Kir could be activated by other phosphoinositides like $\mathrm{PI}(3,4) \mathrm{P}_{2}$ and $\mathrm{PI}(3,4,5) \mathrm{P}_{3}$, which have high structural and chemical similarity to the native agonist $\mathrm{PI}(4,5) \mathrm{P}_{2}$. Both $\mathrm{PI}(3,4) \mathrm{P}_{2}$ and $\mathrm{PI}(3,4,5) \mathrm{P}_{3}$ are phospholipid components of cell membranes involved in many important signal transduction pathways. Logothetis's lab recently utilized electrophysiology techniques to measure the activity of a series of Kir channels, Kir2.1, Kir2.2, K3.4*, and Kir6.2, repetitively, under $\mathrm{PI}(4,5) \mathrm{P}_{2}, \mathrm{PI}(3,4) \mathrm{P}_{2}$ and $\mathrm{PI}(3,4,5) \mathrm{P}_{3} .{ }^{21}$ They discovered that on one extreme $\mathrm{PI}(3,4) \mathrm{P}_{2}$ and $\mathrm{PI}(3,4,5) \mathrm{P}_{3}$ hardly activate the channel (i.e., Kir2.1, a typical Kir channel that requires $\mathrm{PI}(4,5) \mathrm{P}_{2}$ for maintaining normal function) (Group $1)$; and on the other extreme, the current levels induced by $\mathrm{PI}(4,5) \mathrm{P}_{2}, \mathrm{PI}(3,4,5) \mathrm{P}_{3}$, or $\mathrm{PI}(3,4) \mathrm{P}_{2}$ were comparable (i.e., Kir6.2, also sensitive to long chain acyl CoA) (Group 4). In other cases, their responses to $\mathrm{PI}(3,4) \mathrm{P}_{2}$ and $\mathrm{PI}(3,4,5) \mathrm{P}_{3}$ were in-between Kir2.1 and Kir6.2. For example, the mouse Kir2.2 had no response to $\mathrm{PI}(3,4) \mathrm{P}_{2}$, but was partially activated by $\mathrm{PI}(3,4,5) \mathrm{P}_{3}$ (its current reached about $30 \%$ of $\mathrm{PI}(4,5) \mathrm{P}_{2}$ 's) (Group 2). For Kir3.4*, $\mathrm{PI}(3,4) \mathrm{P}_{2}$ activated the current level by $20 \%$ of $\mathrm{PI}(4,5) \mathrm{P}_{2}$, whereas $\mathrm{PI}(3,4,5) \mathrm{P}_{3}$ raised it by $80 \%$ (Group 3). ${ }^{21}$ Thus, each of the thirteen Kir channels could be grouped into one of four subtypes depending on the relative sensitivity to the three PIPs, while they still comprise one large group in that they are all sensitive to $\mathrm{PI}(4,5) \mathrm{P}_{2} .{ }^{21}$ These specific PIP sensitivities indicate that the PIP binding and activation mechanisms are possibly controlled by far more subtle interactions besides the longrange electrostatics, and hence inspire more elaborate investigation on the ligand specificity.

In this study, we apply a rigorous free energy perturbation (FEP) method to model the three PIPs (i.e., $\mathrm{PI}(4,5) \mathrm{P}_{2}, \mathrm{PI}(3,4) \mathrm{P}_{2}$ and $\left.\mathrm{PI}(3,4,5) \mathrm{P}_{3}\right)$ bound upon the chicken Kir2.2 channel to explore the underlying molecular mechanism of these PIPs' specificity. By gradually mutating the native agonist $\mathrm{PI}(4,5) \mathrm{P}_{2}$ to $\mathrm{PI}(3,4,5) \mathrm{P}_{3}$ or $\mathrm{PI}(3,4) \mathrm{P}_{2}$, we measured the free energy changes of each mutant relative to the native ligand by thermodynamic cycles in both bound and free states. The FEP calculation shows a $13.9 \mathrm{kcalmol}^{-1}(\Delta \Delta G)$ and $39.7 \mathrm{kcalmol}^{-1}$ for $\mathrm{PI}(3,4,5) \mathrm{P}_{3}$ and $\mathrm{PI}(3,4) \mathrm{P}_{2}$, respectively, indicating that the variants are less specific to the Kir2.2 channel and confirming the experimental findings on the mouse Kir2.2. ${ }^{21}$ Our results also reveal that the mutation shifted the binding modes from what the native ligand possessed, which was accompanied by the unfavorable solvation environment change around the ligands which eventually affected the channel gating efficiency.

\section{Methods}

\section{Molecular model construction}

The receptor-ligand complex of Kir2.2 and $\mathrm{PI}(4,5) \mathrm{P}_{2}$ was initially configured based on the X-ray crystal structure of a Kir2.2 complexed with a short-chain dioctanoyl derivative of $\mathrm{PIP}_{2}$ (phosphatidylinositol biphosphate; PDB code: $\left.3 \mathrm{SPI}\right) .{ }^{18}$ The missing aliphatic chains of $\mathrm{PI}(4,5) \mathrm{P}_{2}$ molecules as well as missing atoms of the protein were constructed by using the Discovery Studio (Accelrys Inc., San Diego, CA, USA) software. The complete receptor-ligand model was subjected to energy 
minimization using the CHARMM program with an implicit membrane/solvent Generalized Born (GB) model for 1000 steps of steepest descent minimization in order to avoid steric clashes. The receptor-ligand complex was then inserted into a lipid bilayer of 437 1-palmitoyl-2-oleoyl-sn-glycero-3-phosphocholines (POPCs) by using the CHARMM-GUI membrane builder. $^{22}$ The POPC membrane was widely used in the MD simulations for studying $\mathrm{K}$ channels. ${ }^{23-26}$ The final system was prepared after charge neutralization and immersion in $150 \mathrm{mM}$ $\mathrm{KCl}$ solution of $>57000$ TIP3P water molecules, ${ }^{27}$ resulting in a system of $>250000$ atoms in a box of $140 \times 140 \times 160 \AA^{3}$ with the membrane normally aligned on the $z$-axis.

\section{Molecular dynamics simulations}

Inter- and intramolecular potential energies were calculated based on CHARMM36 force field. ${ }^{\mathbf{2 8 , 2 9}}$ The van der Waals interactions were treated with a typical cutoff distance of $12 \AA$, while the long-range electrostatic interactions were estimated with the particle-mesh Ewald method. ${ }^{30}$ Molecular dynamics (MD) simulations were carried out with the NAMD2 software ${ }^{31}$ massively parallelized for IBM Blue Gene ${ }^{32,33}$ with a 2 fs time step in a semi-isotropic isobaric and isothermal (NPT) ensemble of 1 atm and $303.15 \mathrm{~K}$, by which the lateral and perpendicular dimensions of the membrane fluctuate independently. The system was first energy minimized for 10000 steps with several restraints on the POPC membrane in order to retain the cys conformation of the unsaturated bond in the oleoyl chain, the stereochemical configuration (i.e., R-form) of the glycerol linker as well as the membrane planarity. The system was further preequilibrated for $0.5 \mathrm{~ns}$ with a 1 fs time step, as the restraints imposed on the POPC membrane were gradually removed in multiple steps before the production runs. Then, we ran regular molecular dynamics simulations with no further restraints for over $80 \mathrm{~ns}$ with a 2 fs time step.

\section{Free energy perturbation calculation}

The binding affinity changes were calculated by using the alchemical free energy perturbation method. Briefly, the free energy for a given state of a system can be defined with the Helmholtz formula,

$$
G=-k T \ln Z=-k T \ln \left\{\iint \mathrm{d} p \mathrm{~d} q \exp [-\beta H(p, q)]\right\}
$$

where $Z$ and $H(p, q)$ represent the partition function and the Hamiltonian of the system, respectively. The total free energy between two states of interest can be enumerated by summing the fractional free energy changes of multiple small steps from the initial state all the way to the final one through the alchemical mutation,

$$
\Delta G=\sum_{\lambda} \Delta G_{\lambda}
$$

$$
\Delta G_{\lambda}=-k T \ln \langle\exp (-\beta[V(\lambda+\Delta \lambda)-V(\lambda)])\rangle \lambda
$$

Where $V(\lambda)=(1-\lambda) V_{1}+V_{2}$, and $V_{1}$ and $V_{2}$ indicate the potential energies of two end states of our interest. In the alchemical mutation, the parameter $\lambda$ is varied from 0 (state 1 ) to 1 (state 2) along multiple small steps. In our simulations, we employed 21 windows with soft-core potentials (i.e., $\lambda=0,0.00001,0.0001$, $0.001,0.01,0.05,0.1,0.2,0.3,0.4,0.5,0.6,0.7,0.8,0.9,0.95$, $0.99,0.999,0.9999,0.99999,0.999999)$. The $\langle\cdots\rangle_{\lambda}$ represents the ensemble average of the potential energy difference over all available conformations at a given window $\lambda$. In each FEP $\lambda$ window, we obtained 200000 conformations for the ensemble average with a 2 fs time step after the first 4000 steps of equilibration. The current setup provided a reasonable convergence for the binding affinity from our previous tests with larger window sizes and longer simulations. ${ }^{34-37}$ In order to avoid the singularity at the ends (so-called "end-point catastrophe"), the van der Waals potential for perturbed atoms was replaced with a soft-core modified 12-6 Lennard-Jones function, ${ }^{36,37}$ and the electrostatic interactions were switched on (or off) for the appearing (or disappearing) atoms after $\lambda>0.1$ (or $\lambda<0.9$ ) so as for the soft-core potential to repel possible atom overlapping.

In our study, the binding affinity for each non-native ligand (i.e., $\mathrm{PI}(3,4,5) \mathrm{P}_{3}$ or $\left.\mathrm{PI}(3,4) \mathrm{P}_{2}\right)$ was calculated relative to the native agonist $\mathrm{PI}(4,5) \mathrm{P}_{2}$ via the thermodynamic cycle between the bound (i.e., Kir2.2 and ligand) and free states (i.e., ligand only) (Fig. 1b). The initial structure for the bound state was obtained from one of the last snapshots of the MD trajectory with Kir2.2 bound with $\mathrm{PI}(4,5) \mathrm{P}_{2}$ in the POPC membrane. For the free state, we utilized one last snapshot with only $\mathrm{PI}(4,5) \mathrm{P}_{2}$ in the POPC membrane after $20 \mathrm{~ns}$ MD simulation in the same electrolyte solution (i.e., $150 \mathrm{mM} \mathrm{KCl}$ ) as the bound state. For each state, we performed at least 5 independent runs from different initial configurations for better convergence. Thus, at least an $84 \mathrm{~ns}$ (21 windows $\times 0.4 \mathrm{~ns} \times 5$ runs $\times 2$ states) long trajectory was generated for each ligand.

\section{Results and discussion}

In order to investigate the intrinsic binding mode between Kir2.2 and $\mathrm{PI}(4,5) \mathrm{P}_{2}$, we first performed all-atom MD simulation on the Kir2.2-PI(4,5) $\mathrm{P}_{2}$ complex for over $80 \mathrm{~ns}$ (Fig. 1a). The channel complex as well as each subunit remains stable through the entire simulation, as indicated in the stable root mean square deviation (RMSD) fluctuation and consistent root mean square fluctuation (RMSF) patterns (Fig. S1 $\dagger$ ). The overall binding mode is summarized in Fig. $2 \mathrm{a}$ and $\mathrm{b}$. The native agonist, $\operatorname{PI}(4,5) \mathrm{P}_{2}$, maintains its stable position bound to the putative binding site near the interface between the cytoplasmic and transmembrane domains, and between the two adjacent channel subunits. Residue-specific atomic contacts indicate that the residues actively involved in the binding of $\mathrm{PI}(4,5) \mathrm{P}_{2}$ include regions like the slide helix (R65-R78), TM1(W79-A105), TM2 (A158-R186) and tether-helix (P187T193). In particular, several high contacts appear in the middle of the Slide helix to the N-terminus of TM1, and the KKR motif of tether-helix. Site-directed mutagenesis studies confirmed the importance of these residues, indicating that their mutations either kill the channel activity or diminish the channel current drastically. ${ }^{19}$ 

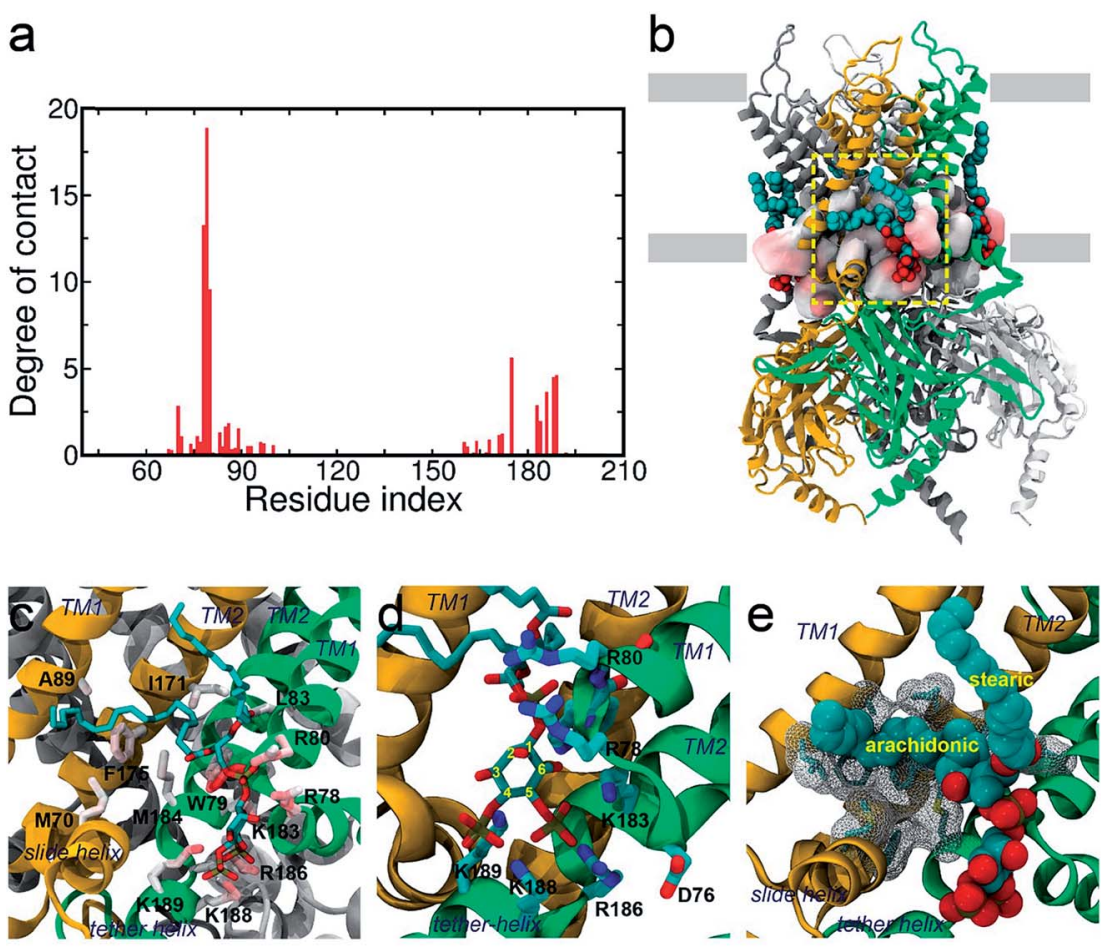

Fig. 2 Binding mode of $\mathrm{PI}(4,5) \mathrm{P}_{2}$ on KIR2.2. (a) Residue-specific atomic contact ratio of $\mathrm{PI}(4,5) \mathrm{P}_{2}$ bound on KIR2.2. (b) A structural snapshot at about $85 \mathrm{~ns}$. PI $(4,5) \mathrm{P}_{2}$ is stably bound at the putative binding sites located on the cytosolic surface of the POPC membrane. (c-e) A detailed binding mode for $\mathrm{PI}(4,5) \mathrm{P}_{2}$ on KIR2.2. The negatively charged head group of $\mathrm{PI}(4,5) \mathrm{P}_{2}$ is stabilized by a multitude of favorable salt-bridge interactions (c and d). The arachidonic chain seems to have favorable interaction with hydrophobic residues, while the stearic chain prefers to interact with lipid molecules (d).

\section{Binding mode of the native agonist $\mathrm{PI}(4,5) \mathrm{P}_{2}$ on Kir2.2}

Binding mode of the inositol head group. The putative binding site of Kir2.2 is characterized by a group of highly conserved positive residues such as R78, R80, K183, R186, K188 and K189. During our simulation, the inositol head group of each $\mathrm{PI}(4,5) \mathrm{P}_{2}$ retained its crystal conformation at the putative binding site (see Fig. 2d). In more detail, the $5^{\prime}$ - and $4^{\prime}$-phosphate groups are both involved in the binding site along with the residues K183, R186, K188 and K189 by favorable saltbridges. This makes the inositol ring parallel to the plane generated by the inner and outer helices, efficiently mediating the helices. Also, this stabilizes the otherwise unstructured Nterminus (i.e., K188 and K189) of the tether helix, tightly holding the CTD toward the TMD, and thus facilitating the open conformation.

Binding mode of the phosphodiester linker. In addition to the specific binding involving the inositol head group, our simulation also reveals the importance of the phosphodiester $1^{\prime}$-phosphate linker of $\mathrm{PI}(4,5) \mathrm{P}_{2}$ and its contribution to the ligand binding stability. Even though the phosphodiester $1^{\prime}$-phosphate is located distantly from the inositol binding site, it is still capable of making a strong interaction with the TMD residues $\mathrm{R} 78, \mathrm{~W} 79$, and $\mathrm{R} 80$ through long-range electrostatic interactions. In fact, these residues are the top three contributors to the binding stability out of all residues in contact with $\mathrm{PI}(4,5) \mathrm{P}_{2}$ (see Fig. 2a). The persistent contacts are largely due to the concerted electrostatic interactions, with two salt-bridges (with R78 and R80) and multiple backbone hydrogen bonds (with R78 and W79) oriented toward the phosphodiester $1^{\prime}$-phosphate linker. Our observations are confirmed by the Xray co-crystallized structure with dioctanoyl glycerol pyrophosphatide acid (PPA), where PPA can bind to this site even without the inositol head group. ${ }^{18}$ This implies that the phosphodiester $1^{\prime}$-phosphate plays a critical role in anchoring $\operatorname{PI}(4,5) \mathrm{P}_{2}$. Therefore, in addition to introducing the inositol head group to the putative binding site, the phosphodiester $1^{\prime}$-phosphate also helps stabilize the binding. Our result also provides an important rationale on the highly conserved residues (i.e., Arg(Lys)Trp-Arg) among the eukaryotic Kirs, although they are not directly involved in channel gating. ${ }^{18}$

Binding mode of acyl chains. Besides the directional interaction through the charged phosphates, it is particularly noteworthy that the lipid acyl chains also appear to interact with Kir2.2 in a specific manner. Intriguingly, the contact analyses reveal that the acyl chains, even with the hydrophobic nature and intrinsic flexibility, seem to have specific contacts with the TMD residues. Such a tendency was more pronounced in the arachidonic chain rather than the stearic one, possibly owing to a different degree of bond saturation. For instance, the fully saturated stearic chain tends to dissolve in the POPC lipid bilayer rather than in contact with the channel. Even when it is in direct contact with the protein, it appears to prefer random contacts. On the other hand, the arachidonic chain with four 
double bonds tends to reside longer near Kir2.2, occupying the hydrophobic groove spanned over the surface composed of the inner and outer helices as well as the interfacial helix from the neighboring subunit (Fig. 2e). Although not as specific as the inositol head or the phosphodiester linker, several hydrophobic hot spots were recognized for the arachidonic chain: M70, L83, L85, F86, A89, I171, I172, F175 and M184. In particular, F175 was found as the highest contacting residue next to the three pivotal residues (i.e., R78, W79 and R80) for the phosphodiester linker.

The saturation state of acyl chains was proposed as an important factor for the channel signaling mechanism. The replacement of the unsaturated arachidonic chain with a saturated one changed the partition of the signaling lipids (i.e., ligand) between the channel protein and lipid raft. ${ }^{38}$ More directly, the chemical diversity of acyl lipids may affect the binding affinity and gating, as shown in the hydrophobic interaction of $\mathrm{PI}(4,5) \mathrm{P}_{2}$ activation of $\mathrm{Ca}_{\mathrm{v}} 2.2 .^{39}$ Our results provide an important insight into the role of acyl chains in stabilizing (or supporting) the ligand binding which can subsequently influence channel gating.

\section{Phosphoinositide specificity for Kir2.2 by free energy perturbation}

Next we turned our focus onto the ligand specificity for Kir2.2 between the native ligand $\mathrm{PI}(4,5) \mathrm{P}_{2}$ and the two common variants $\mathrm{PI}(3,4) \mathrm{P}_{2}$ and $\mathrm{PI}(3,4,5) \mathrm{P}_{3}$. Using FEP, we assessed the binding affinity changes for the mutations from $\mathrm{PI}(4,5) \mathrm{P}_{2}$ to $\mathrm{PI}(3,4,5) \mathrm{P}_{3}$ or to $\mathrm{PI}(3,4) \mathrm{P}_{2}$. Based on the thermodynamic cycle shown in Fig. 1b, the relative binding free energy changes, $\Delta \Delta G$ from $\mathrm{PI}(4,5) \mathrm{P}_{2}$ to $\mathrm{PI}(3,4,5) \mathrm{P}_{3} / \mathrm{PI}(3,4) \mathrm{P}_{2}\left(\Delta \Delta G_{\mathrm{WT} \rightarrow \mathrm{MUT}}\right)$, were obtained by performing multiple FEP simulations for both the bound state $\left(\Delta G_{\text {bound }}\right)$ and free state $\left(\Delta G_{\text {free }}\right)$. Table 1 summarizes the FEP calculation results. For the mutation from $\operatorname{PI}(4,5)$ $\mathrm{P}_{2}$ to $\mathrm{PI}(3,4,5) \mathrm{P}_{3}, \Delta \Delta G=13.9 \mathrm{kcalmol}^{-1}$, whereas for the mutation from $\mathrm{PI}(4,5) \mathrm{P}_{2}$ to $\mathrm{PI}(3,4) \mathrm{P}_{2}, \Delta \Delta G=39.7$ kcalmol $^{-1}$, indicating a strong preference of $\mathrm{PI}(4,5) \mathrm{P}_{2}$ over $\mathrm{PI}(3,4,5) \mathrm{P}_{3}$ or $\mathrm{PI}(3,4) \mathrm{P}_{2}$ (least preferred). Our results are in good agreement with the experimental findings, where $\mathrm{PI}(3,4,5) \mathrm{P}_{3}$ shows only $\sim 30 \%$ activation of Kir2.2 as compared to the wide-type $\operatorname{PI}(4,5)$ $\mathrm{P}_{2}$, while $\mathrm{PI}(3,4) \mathrm{P}_{2}$ shows no activation. ${ }^{21}$
In the normal physiological environment, the inositol head group of $\mathrm{PIP}_{2}$ and $\mathrm{PIP}_{3}$ can hold four and six negative charges, respectively. This implies that the electrostatic interaction might play a critical role in $\mathrm{PI}(4,5) \mathrm{P}_{2}$ binding to Kir2.2. To further explore this, we compared the energetic components of the total binding free energies (Table 1). Expectedly, both mutations showed that the electrostatic component dominates the free energy changes. For example, in the mutation to $\mathrm{PI}(3,4,5) \mathrm{P}_{3}$, the total electrostatic and van der Waals contributions to the binding free energy of $\Delta \Delta G=13.9 \mathrm{kcalmol}^{-1}$ are 13.7 and $2.0 \mathrm{kcalmol}^{-1}$, respectively, with $-1.8 \mathrm{kcalmol}^{-1}$ for the coupling term. Here, we found that the mutation stabilizes the ligand electrostatically, particularly, in the free state, which implies that the strong binding specificity of $\mathrm{PI}(4,5) \mathrm{P}_{2}$ over $\mathrm{PI}(3,4,5) \mathrm{P}_{3}$ originates indirectly from the partitioning difference between the bound and the free states, rather than from destabilization of the ligand in the bound state.

For the mutation from $\mathrm{PI}(4,5) \mathrm{P}_{2}$ to $\mathrm{PI}(3,4) \mathrm{P}_{2}$, similar to that to $\mathrm{PI}(3,4,5) \mathrm{P}_{3}$, it also shows that the total free energy change of $\Delta \Delta G=39.7 \mathrm{kcalmol}^{-1}$ is largely due to the electrostatic free energy change, which is $\Delta \Delta G_{\text {elec }}=40.1 \mathrm{kcalmol}^{-1}$. However, the rationality for the ligand specificity in this case is quite different from the mutation to $\mathrm{PI}(3,4,5) \mathrm{P}_{3}$. Here, the specific binding for $\mathrm{PI}(4,5) \mathrm{P}_{2}$ is directly related to the unfavorable ligand stability in the bound state, as compared to $\mathrm{PI}(3,4) \mathrm{P}_{2}$. In other words, the translocation of one phosphate from $4^{\prime}$ to $3^{\prime}$ position hugely destabilized the binding. Given the equivalent charges between the two ligands, our finding strongly indicates that the binding specificity of $\mathrm{PI}(4,5) \mathrm{P}_{2}$ is regulated by a more sophisticated local binding mode (see below for the details).

\section{Binding mode shift by $\mathrm{PI}(4,5) \mathrm{P}_{2}$ to $\mathrm{PI}(3,4,5) \mathrm{P}_{3}$ mutation}

We next explored the underlying mechanism of the unfavorable free energy changes upon mutations of $\mathrm{PI}(4,5) \mathrm{P}_{2}$ into the nonnative $\mathrm{PI}(3,4) \mathrm{P}_{2}$ and $\mathrm{PI}(3,4,5) \mathrm{P}_{3}$. As described in the aforementioned sections, X-ray crystallography data and MD simulations have indicated that $\mathrm{PI}(4,5) \mathrm{P}_{2}$ interacts with Kir2.2 at the binding site in a sophisticated and well-coordinated way. During the interaction, both $4^{\prime}$ - and $5^{\prime}$-phosphates simultaneously form salt bridges with K188 and K189 (KKR motif on the tether helix). Towards the center of the channel, $5^{\prime}$-phosphate also efficiently

Table 1 FEP results for (a) PIP45 to PIP345 and (b) PIP45 to PIP34

\begin{tabular}{|c|c|c|c|c|c|c|c|c|}
\hline Mutation & $\Delta G$ & $\Delta G_{\text {elec }}{ }^{a}$ & $\Delta G_{\mathrm{vdw}}^{b}$ & $\Delta G_{\text {couple }}^{c}$ & $\Delta G$ & $\Delta G_{\text {elec }}{ }^{a}$ & $\Delta G_{\mathrm{vdw}}^{b}$ & $\Delta G_{\text {couple }}{ }^{c}$ \\
\hline \multirow[t]{2}{*}{ Free } & $-291.5(0.8)$ & $-324.3(0.9)$ & $23.1(0.6)$ & $9.7(0.1)$ & $24.6(1.7)$ & $20.3(1.8)$ & $-12.6(0.4)$ & $17.0(0.4)$ \\
\hline & $\Delta \Delta G^{d, e}$ & $\Delta \Delta G_{\text {elec }}$ & $\Delta \Delta G_{\mathrm{vdW}}$ & $\Delta \Delta G_{\text {couple }}$ & $\Delta \Delta G$ & $\Delta \Delta G_{\text {elec }}$ & $\Delta \Delta G_{\mathrm{vdW}}$ & $\Delta \Delta G_{\text {couple }}$ \\
\hline
\end{tabular}

${ }^{a}$ Electrostatic contribution of free energy. ${ }^{b}$ van der Waals contribution of free energy. ${ }^{c}$ Coupling terms are obtained from $\Delta G_{\text {couple }}=\Delta G-\Delta G_{\text {elec }}-$ $\Delta G_{\mathrm{vdW}} \cdot{ }^{d} \Delta \Delta G=\Delta G_{\text {bound }}-\Delta G_{\text {free }}{ }^{e}$ Standard errors. 
interacts with K183 (in the TM2) and R186. On the other hand, $1^{\prime}$-phosphate, located at the membrane interface, remains anchored by R78, W79 and R80 (the three residues linking the Slide helix and TM1). These residues were reported to be critical in other Kir channels (their mutations would either kill the channel activities or dramatically reduce the ligand sensitivities $\left.{ }^{19}\right)$.

As $\mathrm{PI}(4,5) \mathrm{P}_{2}$ mutates into $\mathrm{PI}(3,4,5) \mathrm{P}_{3}$, the binding pose gets slightly shifted from the native binding configuration albeit without much difference (Fig. $3 \mathrm{c}$ and d). For example, superimposition of a representative chain shows that the inositol phosphates $\mathrm{P} 1, \mathrm{P} 4$ and $\mathrm{P} 5$ of $\mathrm{PI}(3,4,5) \mathrm{P}_{3}$ have been displaced by only $0.9,0.8$ and $1.5 \AA$, respectively, from those of $\mathrm{PI}(4,5) \mathrm{P}_{2}$. Also, a majority of the salt bridges appeared to be well preserved (e.g., K183-P5, R186-P5, and K188-P4). Yet, residue-specific atomic contacts reveal that there are several significant differences between the $\mathrm{PI}(4,5) \mathrm{P}_{2}$ and $\mathrm{PI}(3,4,5) \mathrm{P}_{3}$ complexes (Fig. 3a and Table S1 $\dagger$ ). We highlighted the residues showing remarkable contact change by the residue contact probability difference $\left(\Delta q=q_{\mathrm{MUT}}-q_{\mathrm{WT}}\right)$. The residue-specific atomic contact is defined as the sum of each atomic contact probability of a chosen residue, i.e., $q_{\mathrm{r}}=\sum_{i_{\mathrm{r}}} q_{i_{\mathrm{r}}}$, where the atomic contact probability, $q_{i_{\mathrm{r}}}=\sum_{l} \sum_{m=1}^{4} \delta_{l, m}\left(i_{\mathrm{r}}\right) / \sum_{l} \sum_{m=1}^{4}$, of a heavy atom $i_{\mathrm{r}}$ of a residue $\mathrm{r}$ was obtained by counting the atomic contact $\delta_{l, m}\left(i_{\mathrm{r}}\right)$ with a $5 \AA$ cut-off from any ligand heavy atoms, over all 4 channel subunits and all MD frames (counted by $m$ and $l$ respectively). Taking $|\Delta q|>0.5$ as a criterion, K189, R80, M184 and S87 substantially decreased in the ligand contact by -1.25 , $-0.85,-0.64$ and -0.59 , respectively. Meanwhile, there is a huge increase $(\Delta q=2.50)$ for R78, which is one of the anchoring residues to the phosphodiester linker. In fact, even with one more phosphate, the overall atomic contacts $\left(\Delta q_{\text {all }}=\right.$ $\left.\Delta q_{(-)}+\Delta q_{(+)}=-5.65+4.59=-1.06\right)$ between $\mathrm{PI}(3,4,5) \mathrm{P}_{3}$ and Kir2.2, compared with $\mathrm{PI}(4,5) \mathrm{P}_{2}$, are decreased, implying a weakened connection between $\mathrm{PI}(3,4,5) \mathrm{P}_{3}$ and Kir2.2. This observation is also reflected by the site-directed mutagenesis experiments, which show that neutralizing one of these basic residues decreases the $\mathrm{EC}_{50}$ of $\mathrm{PI}(4,5) \mathrm{P}_{2}$ to Kir2 channels. ${ }^{19}$

As can be seen in Fig. 3 d, the additional phosphate at $3^{\prime}$ position can increase the electrostatic attraction to R78. The interaction is so effective that it accounts for as much as $50 \%$ of the total contact increase. Nonetheless, our simulation showed that the strong attraction between R78 and 3'-phosphate group rather destabilized the ligand binding in at least two different ways. First, it slightly distorted the inositol head group from its native binding conformation. This in turn substantially weakened the salt-bridge between $5^{\prime}$-phosphate and K189, as evidenced in the significant reduction of K189 contact (i.e., $\Delta q=$ $-1.25)$ as well as elongation of the salt-bridge distance. For example, the distance between K189 and 5'-phosphate in one representative structure was found to be elongated from $2.5 \AA$ to $5.7 \AA$ (only heavy atoms counted) after the mutation. Second, it also disturbed the binding of the phosphodiester linker. As $3^{\prime}-$ phosphate begins to interact with R78, the interaction between $1^{\prime}$-phosphate and R80 becomes more unstable, as indicated in the decreased contact (i.e., $\Delta q=-0.85$ ). a

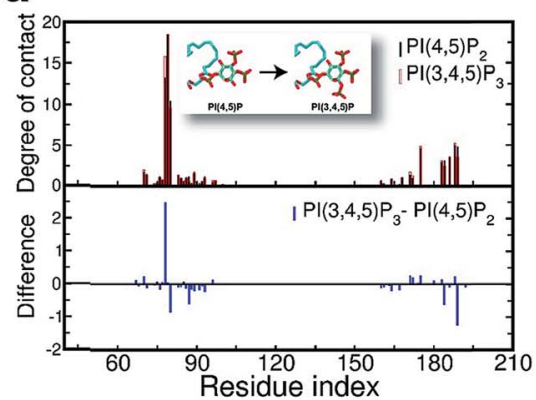

b

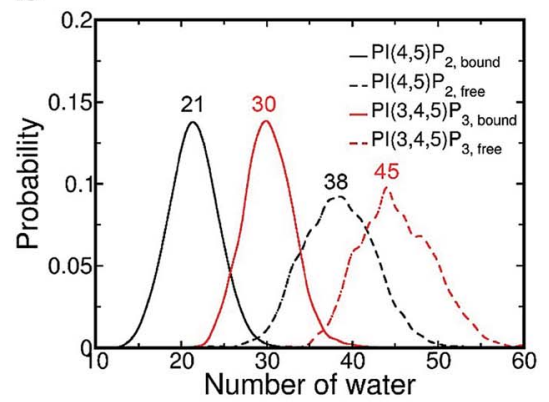

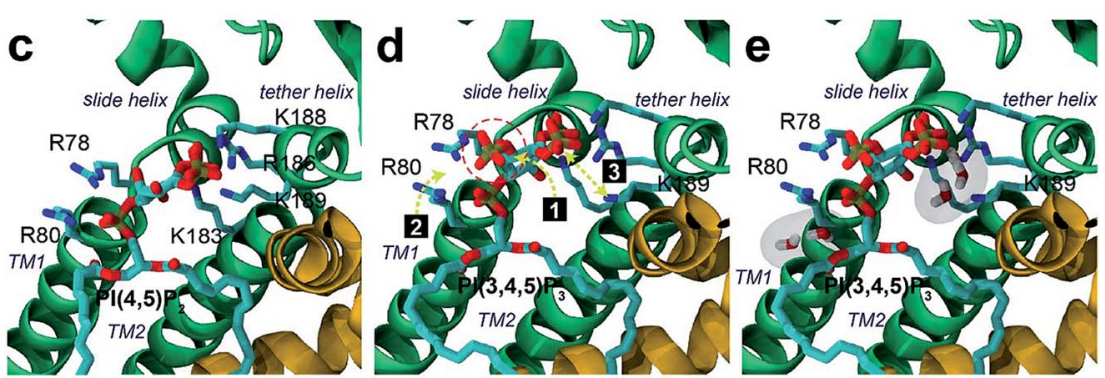

Fig. 3 Binding mode change by mutation from $\mathrm{PI}(4,5) \mathrm{P}_{2}$ to $\mathrm{PI}(3,4,5) \mathrm{P}_{3}$. (a) Residue-specific atomic contact ratio (up) and changes (down) upon the phosphoinositide ligand mutation. (b) Water distribution changes around phosphoinositide ligands. Numbers on each graph indicate average number of water molecules in the first solvation shell. (c) Binding mode of $\mathrm{PI}(4,5) \mathrm{P}_{2}$. (d) Binding mode after mutation to $\mathrm{PI}(3,4,5) \mathrm{P}_{3}$. Number bullets ( 1 to 3 ) describe a schematic order for the binding mode shift upon the mutation. (e) Binding mode shift with $\mathrm{Pl}(3,4,5) \mathrm{P}_{3}$ leaves room for water intrusion near K189 and R80. 
To further investigate the potential impact of key residues on PIPs' binding, we selected two $\mathrm{PI}(4,5) \mathrm{P}_{2}$ binding residues, $\mathrm{K} 189$ and R80, to mutate to alanine, respectively, and conducted the corresponding FEP calculations as the wildtype (Table S3 $\dagger$ ). For the K189A mutant, the free energy changes for $\mathrm{PI}(4,5) \mathrm{P}_{2}$ to $\mathrm{PI}(3,4,5) \mathrm{P}_{3}, \Delta \Delta G_{\mathrm{PIP} 2 \rightarrow \mathrm{PIP} 3}$ is $15.0 \mathrm{kcalmol}^{-1}$; and for the $\mathrm{R} 80 \mathrm{~A}$ mutant, the $\Delta \Delta G_{\mathrm{PIP} 2 \rightarrow \mathrm{PIP} 3}$ is $12.9 \mathrm{kcalmol}^{-1}$. Comparing the wildtype's $\Delta \Delta G_{\mathrm{PIP} 2 \rightarrow \mathrm{PIP} 3}$ of $13.9 \mathrm{kcalmol}^{-1}$, the Lys-deletion from the residue 189 favors $\mathrm{PI}(4,5) \mathrm{P}_{2}$ relatively more than $\mathrm{PI}(3,4,5) \mathrm{P}_{3}$ by about $1 \mathrm{kcalmol}^{-1}$; while the Arg-deletion from the residue 80 reduces the binding affinity of the native ligand $\mathrm{PI}(4,5) \mathrm{P}_{2}$ by about $1 \mathrm{kcalmol}^{-1}$. Although two mutations are, obviously, not sufficient to draw a conclusion, the binding site residues may play a different role in the ligand binding depending on their location and binding contribution. For example, K189 may contribute more to the binding selectivity since it is located near the phosphoinositide head group, while R80 may contribute more to the binding stability due to its role in coordinating to the phosphodiester linker.

Furthermore, we also found that the water environment can be altered with $\mathrm{PI}(3,4,5) \mathrm{P}_{3}$ from that with $\mathrm{PI}(4,5) \mathrm{P}_{2}$. In the free state, on average 38 water molecules were found in the first hydration shell of $\mathrm{PI}(4,5) \mathrm{P}_{2}$, whereas 7 more were found in that of $\mathrm{PI}(3,4,5) \mathrm{P}_{3}$ (i.e., 45 water molecules), possibly due to the additional phosphate group. However, in the bound state, we found 9 more water molecules (i.e., 30 water molecules) around $\mathrm{PI}(3,4,5) \mathrm{P}_{3}$ compared to that of $\mathrm{PI}(4,5) \mathrm{P}_{2}$ (i.e., 21 water molecules). In other words, on average 17 water molecules (38-21) have been desolvated from $\mathrm{PI}(4,5) \mathrm{P}_{2}$ upon binding to Kir2.2, whereas only 15 water molecules $(45-30)$ have been desolvated from $\mathrm{PI}(3,4,5) \mathrm{P}_{3}$. This may indicate that $\mathrm{PI}(3,4,5) \mathrm{P}_{3}$ is in a relatively loose coupling with Kir2.2 despite the increased charge (i.e., $-6 \mathrm{e}$ in the inositol head). In fact, we were able to identify two regions where water can intrude for the case of $\mathrm{PI}(3,4,5) \mathrm{P}_{3}$. One region is found near the inositol head, i.e., a space formed after the salt-bridge breaks between K189 and 5'-phosphate (Fig. 3e). The other was near the phosphodiester linker, i.e., a gap between the lipid bilayer and R80. In addition to water molecules, divalent ions in the local environment may also influence the stability of PIPs by forming stable interactions with phosphate groups, ${ }^{40}$ however, the typical concentration of divalent ions (e.g. $1 \mathrm{mM} \mathrm{MgCl}_{2}$ in electrophysiological experiments) is usually too low to have a meaningful effect on PIPs' binding to Kir channels. In summary, the $3^{\prime}$-phosphate of $\mathrm{PI}(3,4,5) \mathrm{P}_{3}$ introduces a new interaction between $\mathrm{R} 78$ and the inositol head group. However, it can disturb the native binding mode of the inositol head to Kir2.2, especially the salt-bridge between $5^{\prime}$-phosphate and K189. Also, it can perturb the interaction between $1^{\prime}$-phosphate and R80 (Fig. 3d), which effectively weakens the ligand binding accompanied by the loose desolvation, and eventually the gating function for Kir2.2.

\section{Binding mode shift by $\operatorname{PI}(4,5) \mathrm{P}_{2}$ to $\mathrm{PI}(3,4) \mathrm{P}_{2}$ mutation}

Surprisingly, the mutation to $\mathrm{PI}(3,4) \mathrm{P}_{2}$ impacted more dramatically than $\mathrm{PI}(3,4,5) \mathrm{P}_{3}$, on the native binding mode, resulting in a more apparent shift from the native one. For example, the displacements for equivalent phosphates (i.e., P1 and P4) of $\mathrm{PI}(3,4) \mathrm{P}_{2}$ from $\mathrm{PI}(4,5) \mathrm{P}_{2}$ were 2.1 and $1.8 \AA$, respectively, each increased at least $1.0 \AA$ when compared to the corresponding pairs between $\mathrm{PI}(4,5) \mathrm{P}_{2}$ and $\mathrm{PI}(3,4,5) \mathrm{P}_{3}$. Although $\mathrm{PI}(3,4) \mathrm{P}_{2}$, as a configurational isomer of $\mathrm{PI}(4,5) \mathrm{P}_{2}$, has only one phosphate switched from $5^{\prime}$ to $3^{\prime}$ of the inositol ring, the $3^{\prime}$-phosphate was not having strong enough interaction to substitute for that mediated by the $5^{\prime}$-phosphate as in $\mathrm{PI}(4,5) \mathrm{P}_{2}$. More specifically, the distances of K183, R186, and K188 to $5^{\prime}$-phosphate of PI $(4,5)$ $\mathrm{P}_{2}$ were $2.7,2.6$, and $2.6 \AA$, respectively, whereas those to $3^{\prime}$-phosphate of $\mathrm{PI}(3,4) \mathrm{P}_{2}$ have been elongated to 5.6, 7.2, and $4.8 \AA$, respectively. On the other hand, the $3^{\prime}$-phosphate can form a new salt bridge with R78 with a distance of $2.6 \AA$. However, this new single salt-bridge seriously disturbs the stable salt-bridge network, especially the ones formed between $1^{\prime}$-phosphate and R78/R80, and between $4^{\prime}$-phosphate and K188 (e.g., a distance change from 5.6 to $8.4 \AA$ ), thus resulting in a net loss in terms of interaction with the protein.

This change is also reflected in a substantial reduction of contact probabilities (i.e., $\Delta q<-0.5$ ) for a majority of binding site residues, including R80, K183, R186, K188 and K189. Of course, R78 has increased its contact somewhat by $\Delta q=1.19$, similar to the case of $\mathrm{PI}(3,4,5) \mathrm{P}_{3}$, largely due to the new saltbridge from the $3^{\prime}$-phosphate of $\mathrm{PI}(3,4) \mathrm{P}_{2}$ mentioned above. Nevertheless, as expected, the overall residue-contact has been greatly reduced by $\Delta q_{\text {all }}=-6.05\left(=\Delta q_{(+)}+\Delta q_{(-)}=3.26+\right.$ $(-9.31)$ ) from the mutation to $\mathrm{PI}(3,4) \mathrm{P}_{2}$. These findings, together with the above FEP results, indicate serious destabilization in both $\mathrm{PI}(3,4,5) \mathrm{P}_{3}$ and $\mathrm{PI}(3,4) \mathrm{P}_{2}$, but with a significantly more deleterious impact from $\mathrm{PI}(3,4) \mathrm{P}_{2}$.

The larger perturbation was also evidenced by the water distribution changes in the first solvation shell of $\mathrm{PI}(3,4) \mathrm{P}_{2}$. In the free state, $\mathrm{PI}(3,4) \mathrm{P}_{2}$, as a configurational isomer of $\mathrm{PI}(4,5) \mathrm{P}_{2}$, displays more or less similar water distribution with an average of 38 water molecules around the ligand (Fig. 4b). In the bound state, however, more water molecules (also with wider fluctuation) surround $\mathrm{PI}(3,4) \mathrm{P}_{2}$, resulting in a stronger solvation than that observed in the native ligand $\operatorname{PI}(4,5) \mathrm{P}_{2}$. We found that the increased solvation of $\mathrm{PI}(3,4) \mathrm{P}_{2}$ in the bound state was mainly from the deletion of $5^{\prime}$-phosphate. As aforementioned, the deletion removed the important interaction between the phosphate and tether helix, leaving a large space behind. The newly substituted 3-phosphate group tried to compensate for the loss by tilting its binding slightly toward the helix, but it was not sufficient to cause any significant impact. Instead, water molecules entered this space and stabilized the exposed charged residues (i.e., K183, R186 and K188) of the tether helix, as shown in Fig. 4e, where seven water molecules filled in the open space, interacting with the nearby positive residues.

\section{Structural impact on the tether helix}

Furthermore, we noticed that the tether helix loses its helical structure immediately after it loses the salt-bridge interactions with the ligand upon the $5^{\prime}$-phosphate deletion. Comparing Fig. 4c and d, the first helical turn is relaxed to a random coil after the $\mathrm{PI}(4,5) \mathrm{P}_{2}$ to $\mathrm{PI}(3,4) \mathrm{P}_{2}$ mutation. Fig. $4 \mathrm{~d}$ summarizes the 
a

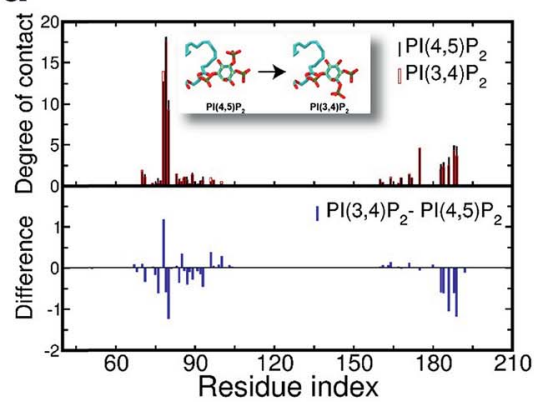

b

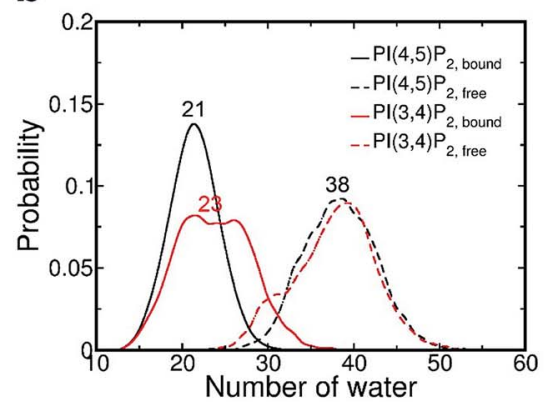

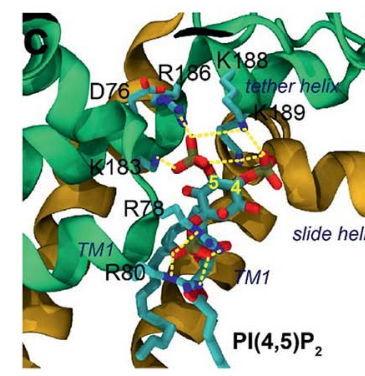
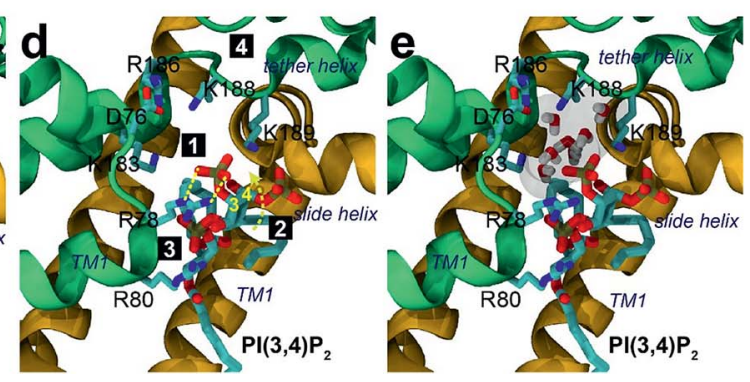

Fig. 4 Binding mode change by mutation from $\mathrm{PI}(4,5) \mathrm{P}_{2}$ to $\mathrm{PI}(3,4) \mathrm{P}_{2}$. (a) Residue-specific atomic contact ratio (up) and changes (down) upon the phosphoinositide ligand mutation. (b) Water distribution changes around phosphoinositide ligands. Numbers on each graph indicate the average number of water molecules in the first solvation shell. (c) Binding mode of $\mathrm{PI}(4,5) \mathrm{P}_{2}$. (d) Binding mode after mutation to $\mathrm{PI}(3,4) \mathrm{P}_{2}$. Number bullets ( 1 to 4 ) describe a schematic order for the binding mode shift upon the mutation. (e) Binding mode shift with $\mathrm{PI}(3,4) \mathrm{P}_{2}$ leaves a large room for water intrusion between the ligand and the positive residues (i.e., K183, R186, and K188) in the binding site. Note that the tether helix has been relaxed with $\mathrm{PI}(3,4) \mathrm{P}_{2}$

binding mode shift along the mutation from $\mathrm{PI}(4,5) \mathrm{P}_{2}$ to $\mathrm{PI}(3,4)$ $\mathrm{P}_{2}$ : (1) P3 group shows up with $5^{\prime}$-phosphate deleted; (2) the inositide group of $\mathrm{PI}(3,4) \mathrm{P}_{2}$ rotates slightly in order to fit the binding site, leaving a large room for water intrusion; (3) new salt bridges formed between R78 and $3^{\prime}$-phosphate; and (4) interactions between $\mathrm{PI}(3,4) \mathrm{P}_{2}$ and K183/R186/K188 are weakened, accompanied by the relaxation of the tether helix to a random coil.

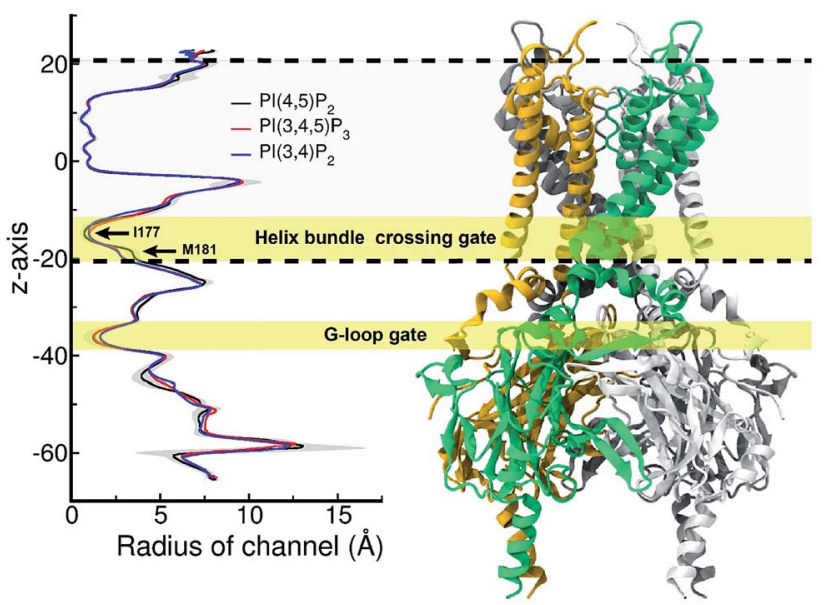

Fig. 5 Pore radius change upon change in the type of phosphoinositide. ore-radii are sensitive to the types of phosphoinositides especially at the helix bundle crossing ( $\mathrm{HBC}$ ) and G-loop gates. In particular, for the HBC gate, the channel near $\mathrm{M} 181$ gets narrowed by $\mathrm{PI}(3,4,5) \mathrm{P}_{3}$ and $\mathrm{PI}(3,4) \mathrm{P}_{2}$, while it is more opened by the wild-type $\mathrm{PI}(4,5) \mathrm{P}_{2}$. The region between two dashed lines indicates TMD of KIR2.2.
Fig. 5 shows the pore radius change upon the mutations to both $\mathrm{PI}(3,4,5) \mathrm{P}_{3}$ and $\mathrm{PI}(3,4) \mathrm{P}_{2}$. As shown in the figure, pore-radii are sensitive to the types of phosphoinositides at the helix bundle crossing (HBC) and G-loop gates. In particular, for the HBC gate, the channel near M181 gets narrowed by $\mathrm{PI}(3,4,5) \mathrm{P}_{3}$ and $\operatorname{PI}(3,4) \mathrm{P}_{2}$, while it is more opened by the wild-type $\mathrm{PI}(4,5) \mathrm{P}_{2}$. Thus, even though no large overall conformational changes were observed in the channel (see Fig. 5), the local interactions especially by the $5^{\prime}$-phosphate group of $\mathrm{PI}(4,5) \mathrm{P}_{2}$ with key residues K183/R186/K188 did play a critical role in maintaining the channel pore size. It is worth noting that $\mathrm{K} 183$ is a highly conserved residue among the Kir family. In Kir2.1, even mutation to arginine might cause channel malfunction, and mutation to glutamine seriously reduces the sensitivity of $\mathrm{PI}(4,5) \mathrm{P}_{2} \cdot{ }^{19}$ Our previous MD simulations on Kir3.1 chimera also showed that K183 tends to form more stable salt bridges with $\mathrm{PI}(4,5) \mathrm{P}_{2}$ in the G-loop open state than in the closed one, suggesting a close relationship between $\mathrm{K} 183$ and $\mathrm{PI}(4,5) \mathrm{P}_{2}$ for the channel opening. ${ }^{15}$ Consistently, absence of interaction with K183 in $\mathrm{PI}(3,4) \mathrm{P}_{2}$ may constitute a reason for its disability to activate the channel. R186 was also experimentally examined in Kir2.1 that affects PIPs' specificity: R186Q mutation is able to recover $\sim 20 \%$ activity of Kir2.1 under $\mathrm{PI}(3,4,5) \mathrm{P}_{3}$, but not under $\mathrm{PI}(3,4)$ $\mathrm{P}_{2}{ }^{21}$ This can be explained by our current simulation. R186, located at the end of TM2, forms a very stable salt-bridge with $5^{\prime}$ phosphate of $\mathrm{PI}(4,5) \mathrm{P}_{2}$ partly by a stable holding for R186 from behind (i.e., via $\varepsilon-\mathrm{N}$ or one of the $\eta$-N's of the guanidinium group) with D76 which is also a conserved residue located in the C-terminus of the Slide helix. In the absence of $5^{\prime}$-phosphate of 
PI(3,4)P2, however, R186 turns toward D76 and forms a strong salt-bridge with D76 (2.9 A between heavy atoms), using two $\eta-N$ 's. It was reported that the opening of channels involves a rotating motion of Slide helix. ${ }^{17,18,41,42}$ The salt bridge of R186-D76 thus might serve as a 'lock' for such rotation. Furthermore, glutamine instead of arginine at this position will lower the energy to unlock, and thus reducing the channel selectivity on PIPs (e.g. Kir6.2).

On the other hand, K188 is the first lysine in the highly conserved KKR motif of the following tether helix in the PIPs' binding region. Many studies have shown conformational changes of the motif (or tether-helix) from the loop to helix along with the opening of the Kir2 channels. $\mathrm{PI}(4,5) \mathrm{P}_{2}$ induces this conformational change and promotes the channel opening. ${ }^{18,42}$ In our simulations we did observe the relaxation of the tether-helix, which is directly related to the loss of a series of important interactions (including with K188 and K189) in the $\mathrm{PI}(3,4) \mathrm{P}_{2}$ complex, as discussed above.

\section{Effect on the channel contact of acyl chains}

Lastly, in addition to the basic residues, some hydrophilic and hydrophobic residues that interact with the acyl chains also show a large decrease of their contact probabilities by mutation to $\mathrm{PI}(3,4,5) \mathrm{P}_{3} / \mathrm{PI}(3,4) \mathrm{P}_{2}$, including $\mathrm{F} 71, \mathrm{~L} 84, \mathrm{~S} 87, \mathrm{~A} 89, \mathrm{~S} 93$ and M184 (Table S1 $\dagger$ ). Most of these residues are mainly located in the TM1, except for F71 in the Slide helix and M184 in the TM2 (see Fig. 2). As discussed before, the arachidonic chain of PI $(4,5)$ $\mathrm{P}_{2}$ can have a relatively stable interaction with the transmembrane helices. Our result indicates that phosphate substitution on the inositide can affect not only the binding mode of the inositol head group contributed mainly by strong and directed electrostatic interaction, but also by the hydrophobic tails through non-specific hydrophobic interaction. Our previous study on the Kir3.1 chimera also indicated that a hydrophobic core, composed of L68, F72 (corresponding to F71 in Kir2.2), V76, L175, F181 and M184 (corresponding to M184 in Kir2.2), helped stabilize the open state of the HBC gate. ${ }^{43}$ According to current results, the arachidonic chain of the native agonist $\mathrm{PI}(4,5) \mathrm{P}_{2}$ also contributed to this hydrophobic core stabilization, while for $\mathrm{PI}(3,4) \mathrm{P}_{2}$ and $\mathrm{PI}(3,4,5) \mathrm{P}_{3}$, their changes on the head group alter their tails' orientations, which further diminishes their stabilization of the open HBC state.

\section{Conclusions}

In this study, we carried out rigorous FEP simulations to investigate the binding events of three PIPs, $\mathrm{PI}(4,5) \mathrm{P}_{2}, \mathrm{PI}(3,4,5)$ $\mathrm{P}_{3}$, and $\mathrm{PI}(3,4) \mathrm{P}_{2}$, at the active site of Kir2.2. By gradually mutating $\mathrm{PI}(4,5) \mathrm{P}_{2}$ to the other two PIPs, we demonstrated a weakened binding affinity to the Kir2.2 channel with $\Delta \Delta G=$ $+13.9 \mathrm{kcalmol}^{-1}$ for $\mathrm{PI}(3,4,5) \mathrm{P}_{3}$ and $+39.7 \mathrm{kcalmol}^{-1}$ for $\mathrm{PI}(3,4)$ $\mathrm{P}_{2}$ respectively, indicating a remarkable energetic advantage of $\mathrm{PI}(4,5) \mathrm{P}_{2}$ in binding with Kir2.2 over the other two PIPs. These results are in good agreement with the previous experimental observations that the $\operatorname{PI}(4,5) \mathrm{P}_{2}$ is the primary agonist to maintain the normal function of Kir2 channels while $\mathrm{PI}(3,4,5) \mathrm{P}_{3}$ and $\mathrm{PI}(3,4) \mathrm{P}_{2}$ hardly activate the channels.
Despite that these three PIPs share very similar chemical and structural properties, they show extremely different specificities in activating Kir2 channels with such dramatic binding affinity differences. Our further analyses revealed the underlying molecular mechanism for those differences. $\mathrm{PI}(4,5) \mathrm{P}_{2}$ is capable of forming a series of critical salt bridges to Kir2.2 with each phosphate specifically corresponding precisely to one or two basic residues (e.g., 1'-phosphate-R78/R80, 4'-phosphate-K188/ K189, 5'-phosphate-R186/K188). However, either adding one more phosphate $\left(\mathrm{PI}(4,5) \mathrm{P}_{2}\right.$ to $\left.\mathrm{PI}(3,4,5) \mathrm{P}_{3}\right)$ or changing the positions of phosphate $\left(\mathrm{PI}(4,5) \mathrm{P}_{2}\right.$ to $\left.\mathrm{PI}(3,4) \mathrm{P}_{2}\right)$ cannot accurately reproduce these interactions, resulting in a substantial reduction of several critical interactions. Furthermore, the destabilization of $\mathrm{PI}(3,4) \mathrm{P}_{2}$ was found to originate mainly from the unstable bound state, whereas $\mathrm{PI}(3,4,5) \mathrm{P}_{3}$ binding is mostly affected by a more preferred partition for the free state. In addition to strong electrostatic interactions, we found that the arachidonic chain of $\operatorname{PI}(4,5) \mathrm{P}_{2}$ also contributes to the lipid agonism via favorable hydrophobic core formation with nearby hydrophobic residues such as M70, L83, L85, F86, A89, I171, I172, F175 and M184.

Finally, it should be noted that previous studies have shown that $\mathrm{PI}(4,5) \mathrm{P}_{2}$ controls the channel gates through allosteric regulation. It is known that the $\mathrm{PI}(4,5) \mathrm{P}_{2}$ binding causes conformational changes of Kir2.2, such as a new helix formation in the $\mathrm{N}$-terminal Slide helix from the disordered fragment, and another helix formation in the tether-helix from a loop, which is observed in our current simulation as well. ${ }^{18}$ However, a detailed molecular picture by which how $\mathrm{PI}(4,5) \mathrm{P}_{2}$ binding can couple/regulate the Kir2 channel gating is still not available, which needs further studies in the future. Uncovering such a coupling mechanism would offer deeper insights to further interpret the specificity of PIPs to Kir channels. Finally, it should be mentioned that FEP can converge slowly particularly on systems where the environments of the target alchemical modification undergo slow response fluctuations; for this purpose, various advanced sampling strategies, such as Orthogonal Space Random Walk (OSRW), were developed $^{\mathbf{4 4}}$ to help reduce the enormous computational resources required and better understand the underlying mechanism of the PIP's specificity for potassium channels.

\section{Conflicts of interest}

There are no conflicts to declare.

\section{Acknowledgements}

This work was partially supported by the National Natural Science Foundation of China under Grant No. 11574224, 21503140, 11374221, and 21503140. RZ acknowledges the support from IBM Blue Gene Science Program (W1258591, W1464125, W1464164).

\section{References}

1 D. W. Hilgemann, Cytoplasmic ATP-dependent regulation of ion transporters and channels: mechanisms and messengers, Annu. Rev. Physiol., 1997, 59, 193-220. 
2 D. W. Hilgemann, S. Feng and C. Nasuhoglu, The complex and intriguing lives of PIP2 with ion channels and transporters, Sci. Signaling, 2001, 2001(111), re19.

3 M. Takano and S. Kuratomi, Regulation of cardiac inwardly rectifying potassium channels by membrane lipid metabolism, Prog.Biophys. Mol. Biol., 2003, 81(1), 67-79.

4 B. C. Suh and B. Hille, Regulation of ion channels by phosphatidylinositol 4,5-bisphosphate, Curr.Opin.Neurobiol., 2005, 15(3), 370-378.

5 L. H. Xie, S. A. John, B. Ribalet and J. N. Weiss, Activation of inwardly rectifying potassium (Kir) channels by phosphatidylinositol-4,5-bisphosphate (PIP2): interaction with other regulatory ligands, Prog.Biophys. Mol. Biol., 2007, 94(3), 320-335.

6 C. L. Huang, Complex roles of PIP2 in the regulation of ion channels and transporters, Am. J. Physiol. Renal.Physiol., 2007, 293(6), F1761-F1765.

7 N. Gamper and M. S. Shapiro, Regulation of ion transport proteins by membrane phosphoinositides, Nat. Rev. Neurosci., 2007, 8(12), 921-934.

8 A. Rosenhouse-Dantsker and D. E. Logothetis, Molecular characteristics of phosphoinositide binding, Pfluegers Arch., 2007, 455(1), 45-53.

9 D. E. Logothetis, D. Lupyan and A. Rosenhouse-Dantsker, Diverse Kir modulators act in close proximity to residues implicated in phosphoinositide binding, J. Physiol., 2007, 582(3), 953-965.

10 S. J. Tucker and T. Baukrowitz, How highly charged anionic lipids bind and regulate ion channels, J. Gen. Physiol., 2008, 131(5), 431-438.

11 B. C. Suh and B. Hille, PIP2 is a necessary cofactor for ion channel function: how and why?, Annu. Rev. Biophys., 2008, 37, 175-195.

12 D. W. Hilgemann and R. Ball, Regulation of cardiac $\mathrm{Na}^{+}$, $\mathrm{Ca}^{2+}$ exchange and KATP potassium channels by PIP2, Science, 1996, 273(5277), 956.

13 S.-L. Shyng and C. G. Nichols, Membrane Phospholipid Control of Nucleotide Sensitivity of KATP Channels, Science, 1998, 282(5391), 1138-1141.

14 C.-L. Huang, S. Feng and D. W. Hilgemann, Direct activation of inward rectifier potassium channels by PIP2 and its stabilization by G[beta][gamma], Nature, 1998, 391(6669), 803-806.

15 X.-Y. Meng, H.-X. Zhang, E. Logothetis Diomedes and M. Cui, The Molecular Mechanism by which PIP2 Opens the Intracellular G-Loop Gate of a Kir3.1 Channel, Biophys. J., 2012, 102(9), 2049-2059.

16 S.-J. Lee, et al., Secondary anionic phospholipid binding site and gating mechanism in Kir2.1 inward rectifier channels, Nat. Commun., 2013, 4, 2786.

17 Â. R. Whorton Matthew and R. MacKinnon, Crystal Structure of the Mammalian GIRK2 $\mathrm{K}^{+}$Channel and Gating Regulation by G Proteins, PIP2, and Sodium, Cell, 2011, 147(1), 199-208.

18 S. B. Hansen, X. Tao and R. MacKinnon, Structural basis of PIP2 activation of the classical inward rectifier $\mathrm{K}^{+}$channel Kir2.2, Nature, 2011, 477(7365), 495-498.
19 C. M. B. Lopes, et al., Alterations in Conserved Kir ChannelPIP2 Interactions Underlie Channelopathies, Neuron, 2002, 34(6), 933-944.

20 D. E. Logothetis, T. Jin, D. Lupyan and A. RosenhouseDantsker, Phosphoinositide-mediated gating of inwardly rectifying $\mathrm{K}(+)$ channels, Pfluegers Arch., 2007, 455(1), 83-95.

$21 \mathrm{~T}$. Rohacs, et al., Specificity of activation by phosphoinositides determines lipid regulation of Kir channels, Proc. Natl. Acad. Sci. U. S. A., 2003, 100(2), 745-750.

22 S. Jo, T. Kim, V. G. Iyer and W. Im, CHARMM-GUI: a web-based graphical user interface for CHARMM, $J$. Comput. Chem., 2008, 29(11), 1859-1865.

23 D. A. Köpfer, et al., Ion permeation in $\mathrm{K}^{+}$channels occurs by direct Coulomb knock-on, Science, 2014, 346(6207), 352-355.

24 M. Ø. Jensen, et al., Mechanism of Voltage Gating in Potassium Channels, Science, 2012, 336(6078), 229-233.

25 L. Delemotte, M. Tarek, M. L. Klein, C. Amaral and W. Treptow, Intermediate states of the Kv1.2 voltage sensor from atomistic molecular dynamics simulations, Proc. Natl. Acad. Sci. U. S. A., 2011, 108(15), 6109-6114.

26 Z. Gu, et al., Exploring the Nanotoxicology of $\mathrm{MoS}_{2}$ : A Study on the Interaction of $\mathrm{MoS}_{2}$ Nanoflakes and $\mathrm{K}^{+}$Channels, ACS Nano, 2018, 12(1), 705-717.

27 W. L. Jorgensen, J. Chandrasekhar, J. D. Madura, R. W. Impey and M. L. Klein, Comparison of simple potential functions for simulating liquid water, J. Chem. Phys., 1983, 79, 926-935.

28 R. B. Best, et al., Optimization of the additive CHARMM allatom protein force field targeting improved sampling of the backbone $\phi, \psi$ and side-chain $\chi 1$ and $\chi 2$ dihedral angles, $J$. Chem. Theory Comput., 2012, 8(9), 3257-3273.

29 J. B. Klauda, et al., Update of the CHARMM all-atom additive force field for lipids: validation on six lipid types, J. Phys. Chem. B, 2010, 114(23), 7830-7843.

30 T. A. Darden, D. M. York and L. G. Pedersen, Particle mesh Ewald: An NlogN method for Ewald sums in large systems, J. Chem. Phys., 1993, 98, 10089-10092.

$31 \mathrm{~J}$. C. Phillips, et al., Scalable molecular dynamics with NAMD, J. Comput. Chem., 2005, 26(16), 1781-1802.

32 S. Kumar, et al., Scalable Molecular Dynamics with NAMD on Blue Gene/L, IBM J. Res. Dev., 2008, 52, 177-188.

33 B. G. Fitch, et al., Blue Matter: Strong scaling of molecular dynamics on Blue Gene/L, Springer Berlin Heidelberg, 2006.

34 S.-g. Kang, et al., Molecular Recognition of Metabotropic Glutamate Receptor Type 1 (mGluR1): Synergistic Understanding with Free Energy Perturbation and Linear Response Modeling, J. Phys. Chem. B, 2014, 118(24), 63936404.

35 Z. Xia, T. Huynh, S.-g Kang and R. Zhou, Free-energy simulations reveal that both hydrophobic and polar interactions are important for influenza hemagglutinin antibody binding, Biophys. J., 2012, 102(6), 1453-1461.

36 T. C. Beutler, A. E. Mark, R. C. van Schaik, P. R. Gerber and W. F. van Gunsteren, Avoiding singularities and numerical instabilities in free energy calculations based on molecular simulations, Chem. Phys. Lett., 1994, 222(222), 529-539. 
37 M. Zacharias, T. P. Straatsma and J. A. McCammon, Separation-Shifted Scaling, a New Scaling Method for Lennard-Jones Interactions in Thermodynamic Integration, J. Chem. Phys., 1994, 100(12), 9025-9031.

38 A. Schmidt, et al., Endophilin I mediates synaptic vesicle formation by transfer of arachidonate to lysophosphatidic acid, Nature, 1999, 401(6749), 133-141.

39 B. Hille, E. J. Dickson, M. Kruse, O. Vivas and B. C. Suh, Phosphoinositides regulate ion channels, Biochim.Biophys. Acta, 2015, 1851(6), 844-856.

40 B. Luan, K. L. Chen and R. Zhou, Mechanism of DivalentIon-Induced Charge Inversion of Bacterial Membranes, $J$. Phys. Chem. Lett., 2016, 7(13), 2434-2438.
41 O. B. Clarke, et al., Domain Reorientation and Rotation of an Intracellular Assembly Regulate Conduction in Kir Potassium Channels, Cell, 2010, 141(6), 1018-1029.

42 H.-L. An, et al., The Cytosolic GH Loop Regulates the Phosphatidylinositol 4,5-Bisphosphate-induced Gating Kinetics of Kir2 Channels, J. Biol. Chem., 2012, 287(50), $42278-42287$.

43 X.-Y. Meng, S. Liu, M. Cui, R. Zhou and D. E. Logothetis, The Molecular Mechanism of Opening the Helix Bundle Crossing (HBC) Gate of a Kir Channel, Sci. Rep., 2016, 6, 29399.

44 L. Zheng, M. Chen and W. Yang, Random walk in orthogonalspace to achieve efficient free-energy simulation of complex systems, Proc. Natl. Acad. Sci. U. S. A., 2008, 105(51), 20227-20232. 